\title{
Caracterización de resinas epoxi para uso en conectores de ductos flexibles
}

\author{
Characterization of epoxy resins for \\ use in end fittings of flexible pipes
}

Facundo Javier Wedekamper ${ }^{1}$, Diego Andrés Lorio ${ }^{1}$, Telmo Roberto Strohaecker ${ }^{1,2}$

\author{
${ }^{1}$ Programa de Pós-Graduação em Engenharia de Minas, Metalúrgica e de Materiais, UFRGS, CEP: 91501-970, Porto \\ Alegre, Rio Grande do Sul - RS, Brasil. \\ e-mail: facundowedekamper@gmail.com; loriodiegoandres@gmail.com \\ ${ }^{2}$ Departamento de Metalurgia, Escola de Engenharia, UFRGS, CEP: 90035-190, Porto Alegre, Rio Grande do Sul - RS, \\ Brasil \\ e-mail: telmo@demet.ufrgs.br
}

\section{RESUMEN}

En la producción offshore de gas y petróleo se utilizan con frecuencia ductos flexibles, los conectores son la conexión entre el cuerpo de la línea flexible y las unidades de producción. En el montaje de este componente se utiliza resina epoxi para llenar el espacio libre en su el interior. Este material polimérico fija los alambres de la armadura de tracción del ducto flexible dentro del conector, formando el sistema de anclaje. Un punto crítico en el avance de la industria petrolífera, más allá de la frontera tecnológica de la explotación offshore, es el desempeño estructural de los conectores. Por lo que el material epóxido utilizado en este componente crítico debe tener las características adecuadas que le permita resistir las solicitaciones mecánicas. En este trabajo se presenta una caracterización de cinco resinas epoxis diferentes con el objetivo de comparar sus propiedades y determinar su aptitud para ser aplicadas en conectores. Ensayos de arrancamiento, compresión, cizalla y dureza, fueron realizados a fin de evaluar el desempeño de estos materiales para dicha aplicación. El desempeño de las cinco resinas fue comparada a través de un análisis TOPSIS, en esta técnica se valoran los criterios a través de su similitud con la solución ideal y se pondera la influencia de cada parámetro o propiedad según su relevancia en el sistema de anclaje.

Las resinas analizadas mostraron valores similares de dureza, sin embargo, los valores de tensión compresiva en el límite elástico y en la ruptura, así como la tensión máxima de cizalla evidenciaron diferencias mayores al 30\%. A su vez, los resultados de los ensayos de arrancamiento demostraron diferencias aún más significativas en los parámetros calculados. Finalmente la resina más adecuada para la aplicación fue seleccionada a través del análisis TOPSIS.

Palabras clave: Epoxi, Ensayos de Arrancamiento, Conector, Ductos Flexibles.

\section{ABSTRACT}

In the offshore productions of gas and oil are frequently used flexible pipes. The end fitting is the connection between the flexible pipe body and the production unit, at the assembly of this component is used epoxy resin to fill the free space inside it. This polymeric material holds the tensile armor wires to form the anchoring system. A critical point in the oil industry advance, beyond the technological frontier of offshore oil extraction, is the structural performance of the end fittings. In this critical component, the epoxy material must have the appropriate characteristics that allow resist the mechanical solicitations. This paper presents a characterization of five epoxy materials with the intention to determine their ability to be applied at end fittings. Pull out tests, compression tests, shear testes and measurement of hardness, were made to value the performance of those materials for this application. The performance of the five resins was compared through a TOPSIS analysis, this technique criteria are valued by their similarity to ideal solution and the influence of each parameter or property is weighted according to their importance in the system of anchoring.

Resins tested showed similar hardness values, however, the values of compressive stress at yield and com- 
pressive stress at break, and the maximum shear stress showed $30 \%$ greater differences. Furthermore, the results of pull out tests demonstrated significant differences for the calculated parameters. Finally, the resin more suitable for application on end fittings was selected by analyzing TOPSIS.

Keywords: Epoxy, Pull out Test, End Fitting, Flexible Pipe.

\section{INTRODUCCIÓN}

La constante expansión hacia nuevas fronteras en la producción offshore de hidrocarburos conduce a una creciente demanda de ductos flexibles. Esto se debe principalmente a las ventajas que estos ductos tienen frente a otros sistemas en los complejos arreglos submarinos utilizados para extracción de petróleo y gas en yacimientos ubicados en aguas profundas y ultra-profundas [1]. La estructura de los ductos flexibles está formada principalmente por camadas cilíndricas de polímeros y arreglos helicoidales de metal, cada una de estas camadas tiene características específicas y cumple una función determinada.

En los extremos de las líneas flexibles, son instalados los conectores, estos son el vínculo entre el cuerpo del ducto y las unidades de producción. De acuerdo con la norma API 17B [2] se destacan dos propósitos para este elemento: 1) Su construcción debe ser de tal forma que se transmitan las cargas axiales y momentos de flexión del ducto sin damnificar las camadas que transportan el fluido y 2) proporcionar una adecuada transición de la presión entre el ducto y el cuerpo del conector.

Cuando se realiza la operación de montaje de los conectores se aplica resina epoxi para rellenar el espacio libre en su interior. Después del proceso de cura, este material polimérico fija los alambres de acero que constituyen la armadura de tracción del ducto flexible dentro del conector, formando el denominado sistema de anclaje.

Las características de las resinas epoxi que se destacan en cuanto a la aplicación para conectores son: su alta resistencia mecánica, sus cualidades como adhesivo y su estabilidad frente a diluyentes. Estas resinas se clasifican como polímeros termoestables, contienen dentro de su molécula por lo menos dos grupos terminales del tipo epoxi y se caracterizan por formar una red polimérica tridimensional (reticulada) [3-5].

La creciente exploración y explotación de campos petrolíferos offshore ubicados en aguas profundas y ultra-profundas, como los localizados en la región brasileña del pres-sal, impone nuevos desafíos técnicos y tecnológicos a la industria, esto es en gran medida por las difíciles condiciones ambientales, las grandes profundidades de lámina de agua y altos diferenciales de presión que se presentan [1]. Desafíos que se deben afrontar en los proyectos de diseño de los conectores. En este trabajo se presenta una caracterización de cinco resinas epoxis comerciales, con el objetivo de comparar ciertas propiedades y determinar su aptitud para ser aplicadas en conectores.

\section{MATERIALES Y MÉTODOS}

\subsection{Delineamiento experimental}

Para caracterizar las resinas se propuso ensayos de compresión y cizalla, indicados por la norma ISO 14628-2 [6] para materiales del tipo epoxi utilizados en conectores. Adicionalmente se realizaron mediciones de dureza en la escala Shore D.

Una de las características más importantes de las resinas epoxis para la aplicación en conectores, es la adhesión con los alambres de acero que forman la armadura de tracción de los ductos flexibles, siendo éste un factor determinante en la performance del sistema de anclaje. En el estudio de DAFLON [4] se destaca que la adhesión se ve influenciada principalmente por las características físico-químicas de los materiales que componen la junta, haciendo mención a la tensión superficial del adhesivo y el sustrato, la polaridad, la rugosidad y la interface. TORRES et al. [7], así como otros autores [4,8] proponen ensayos de arrancamiento como una forma simplificada de evaluar el sistema de anclaje. En este ensayo, un trozo de alambre de acero recto es embebido en resina epoxi, después del curado de la resina se aplica una carga tractiva al alambre arrancándolo del material polimérico.

\subsection{Preparación de muestras}

Para este trabajo se evaluaron 5 sistemas epoxis bicomponentes (resina/endurecedor): SW404/HY404, AW4804/HW4804 y XAW1395/HY950 de la marca Huntsman, THERMOSET N³00/N65 y THERMOSET EP-20/N¹8 de la marca comercial Lord. Estos sistemas son denominados en este texto como "A", "B", 
"C", "D" y "E" respectivamente en este texto.

Las probetas fueron moldeadas de acuerdo a cada tipo de ensayo utilizando moldes de aluminio. En la mezcla se utilizaron las proporciones de resina/endurecedor recomendadas por el fabricante. El curado de las probetas se realizó en un recinto cerrado a temperatura constante durante un período de tiempo de $24 \mathrm{hs}$.

Para los ensayos de compresión se confeccionaron probetas cilíndricas de 12,7 mm de diámetro y 25 $\mathrm{mm}$ de longitud como se ilustra en la Figura 3.a. Las dimensiones de las probetas referentes a los a los ensayos de cizalla fueron de 50x50x12,7 mm como se observa de forma ejemplificada en la Figura 3.b. Finalmente la manufactura de las probetas para los ensayos de arrancamiento se realizó utilizando trozos de alambre rectos de armadura de tracción, con dimensiones en la sección transversal de 2,5x5 mm, los cuales fueron embebidos dentro de un cilindro de resina epoxi de $30 \mathrm{~mm}$ de diámetro y de $65 \mathrm{~mm}$ de longitud, sobresaliendo sección de alambre por ambos extremos del cilindro (Figura 3.c).

a)

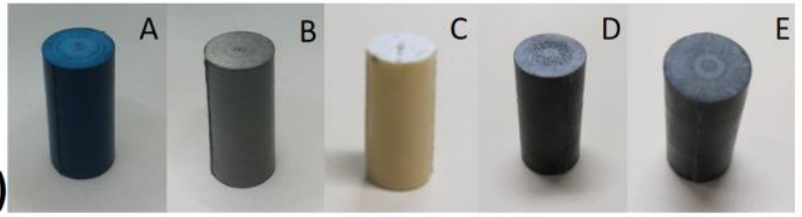

b)

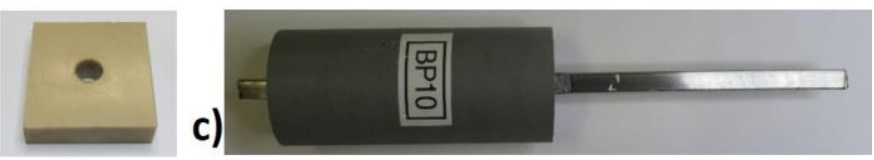

Figura 1: Probetas para ensayos de: a) compresión, b) cizalla y c) arrancamiento.

\subsection{Ensayos de Compresión, cizalla, arrancamiento y medición de dureza}

Los ensayos se realizaron en ambientes con temperatura controlada de $23^{\circ} \mathrm{C}$, bajo las siguientes condiciones:

1. Compresión: un total de 6 muestras de cada tipo de resina evaluada, fueron ensayadas a la compresión utilizando un equipamiento INSTRON 5585 , a la velocidad de $1,3 \mathrm{~mm} / \mathrm{min}$, siguiendo la norma ASTM D695-10 [9]. A partir de este tipo de ensayos se analizaron los valores de tensión compresiva en el límite elástico y en la ruptura.

2. Cizalla: los ensayos de cizalla se realizaron de acuerdo con la norma ASTM D732-02 [100] en un pórtico MTS Landmark 370. Siendo ensayadas 5 muestras por tipo de resina epoxi, a una velocidad de $1,25 \mathrm{~mm} / \mathrm{min}$. El parámetro analizado en este ensayo fue la tensión máxima de cizalla.

3. Arrancamiento: para los ensayos de arrancamiento se utilizó un pórtico MTS Landmark 370 con una garra hidráulica para sujetar un extremo de la muestra y un dispositivo especial de agarre hecho en acero con el fin de sujetar el otro extremo. Se ensayaron 12 muestras de cada tipo de resina a una velocidad de $1 \mathrm{~mm} / \mathrm{min}$.

4. Medición de dureza: las mediciones de dureza fueron realizadas en la escala Shore D sobre uno de los laterales de las probetas de cizalla y fue utilizado un durómetro TM-TH210 en una base para aplicar carga constante, se realizaron 5 mediciones para cada tipo de resina evaluada.
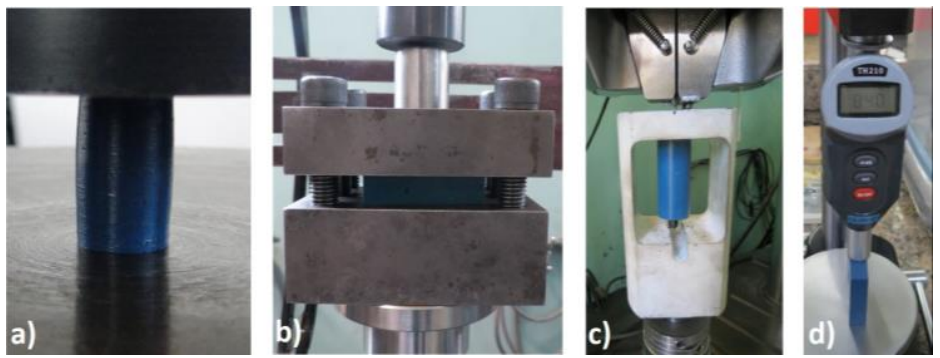

Figura 2: Ensayos de: a) compresión, b) cizalla, c) arrancamiento y d) medición de dureza.

\subsection{Análisis de datos de los ensayos de arrancamiento}

Para evaluar los ensayos de arrancamiento y comparar los resultados entre sí, se construyeron curvas como la 
que ejemplifica la Figura 5, el eje de las abscisas representa el desplazamiento durante el ensayo, y el eje de las ordenadas representa la tensión de cizallamiento que tiende a separar la junta formada entre el alambre de la armadura de tracción y la resina epoxi. Este valor de tensión se obtiene al dividir la fuerza tractiva aplicada por el área de interface entre la resina epoxi y el alambre de acero. Las curvas obtenidas en los ensayos de arrancamiento son similares a las presentadas en el trabajo de CAMPELLO [8] donde se pueden diferenciar dos etapas. En la primera etapa la junta formada por el acero y la resina permanece adherida hasta un valor máximo (en este texto es denominado como tensión por adhesión), en esta fase no se produce desplazamiento relativo entre los materiales de la junta. Luego en la segunda etapa la carga cae bruscamente para mantenerse en un valor casi constante, donde se presenta la fricción entre el alambre y la resina con desplazamiento relativo entre ambos materiales. Estos dos parámetros característicos fueron determinados a partir de los resultados de los ensayos de arrancamiento.

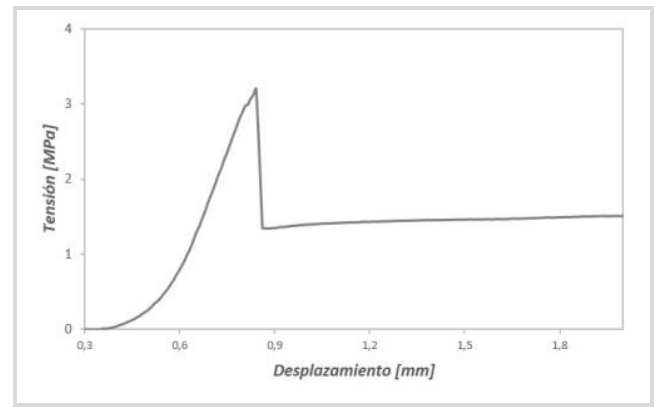

Figura 3: Curva típica de ensayo de arrancamiento.

\subsection{Evaluación y comparación entre resinas}

Para valorar la performance de cada resina epoxi en referencia a las demás resinas evaluadas, en cuanto a su aplicación en sistemas de anclaje para conectores de ductos flexibles, se utilizó la técnica de análisis TOPSIS (Technique for Order of Preference by Similarity to Ideal Solution). Este es un método análisis que permite valorar varios criterios simultáneamente y compararlos a través de su similitud con la solución ideal [11]. La solución ideal en todos los criterios que se contemplaron en este estudio, es la que maximiza los valores de los parámetros obtenidos. La técnica TOPSIS requiere de una ponderación de los criterios según su relevancia en el análisis, por lo que los parámetros obtenidos a partir de los ensayos y mediciones realizadas se valoraron de acuerdo con su importancia relativa para un proyecto de desarrollo del sistema de anclaje para conectores de ductos flexibles. Esta valoración se observa en la

Tabla 1 y se realizó basándose en revisiones bibliográficas y consultas técnicas a personal experimentado [7, $8,12-14]$.

Tabla 1: Ponderación de parámetros TOPSIS

\begin{tabular}{c|c|c}
\hline PARÁMETRO & PONDERACIÓN & PONDERACIÓN RELATIVA \\
\hline Tensión en fricción en arrancamiento & 5 & 0,263 \\
\hline Máxima tensión de adhesión en arrancamiento & 4 & 0,211 \\
\hline Tensión compresiva para la ruptura & 4 & 0,211 \\
\hline Tensión compresiva para el límite elástico & 3 & 0,158 \\
\hline Tensión de cizalla & 2 & 0,105 \\
\hline Dureza & 1 & 0,053 \\
\hline \multicolumn{2}{c|}{ Sumatoria } & 1 \\
\hline
\end{tabular}

\section{RESULTADOS}

En la Figura 4 pueden observarse los valores medios y el desvío estándar de la tensión compresiva en el límite elástico y la tensión compresiva para la ruptura. La resina A muestra el mayor valor de límite elástico, sin embargo la resina B presenta un valor sobresaliente de tensión a la ruptura comparando a las demás. En el gráfico se observa que el mayor valor de tensión en el límite elástico, difiere del menor un $32 \%$ y diferencias entre valores de tensión a la ruptura son aún mayores, llegando hasta $57 \%$. 


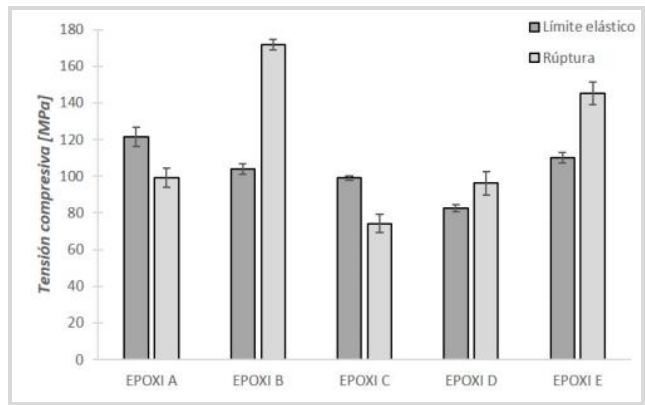

Figura 4: Ensayos de compresión.

Los resultados obtenidos en los ensayos de cizalla se resumen en el gráfico de la Figura 5, donde se ven los valores medios de la máxima tensión de cizalla y sus desvíos estándar. En el gráfico puede observarse que la resina A presenta el mejor desempeño en comparación a las demás, si se compara el mayor valor obtenido con el menor, la diferencia es de $32 \%$ aproximadamente.

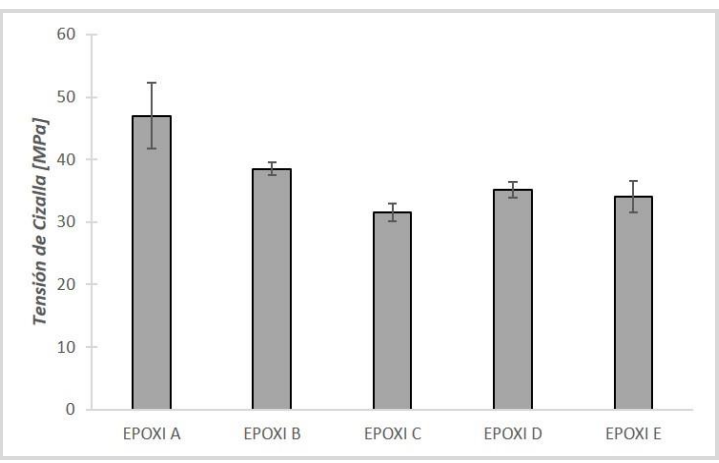

Figura 5: Ensayos de cizalla.

Los valores medios y los desvíos estándar calculados a partir de los resultados de los ensayos de arrancamiento son expuestos en la Figura 6, en el cual se presentan tanto la tensión máxima en la fase de adhesión del ensayo, como la tensión en la región donde aparece la fricción entre la resina epoxi y el acero de la armadura de tracción. En el gráfico se destaca que la junta acero/resina formada por el material C muestra una tensión 51,55\% más elevada que la inmediata anterior, mientras no se produce desplazamiento relativo entre materiales. La variación máxima entre valores medios en esta fase del ensayo es del 76,93\%; mientras que la mayor variación entre medias, una vez que se presenta el desplazamiento relativo entre materiales, es de $69,24 \%$.

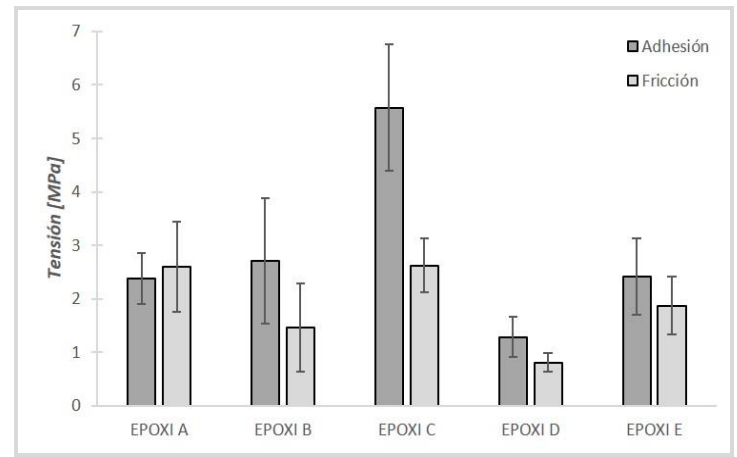

Figura 6: Ensayos de arrancamiento.

Los valores medios de las mediciones de dureza en la escala Shore D se observan en el gráfico de la Figura 7. Es posible observar que las diferentes resinas presentan poca diferencia de dureza entre ellas, la mayor diferencia de los valores medios es de $4 \%$. 


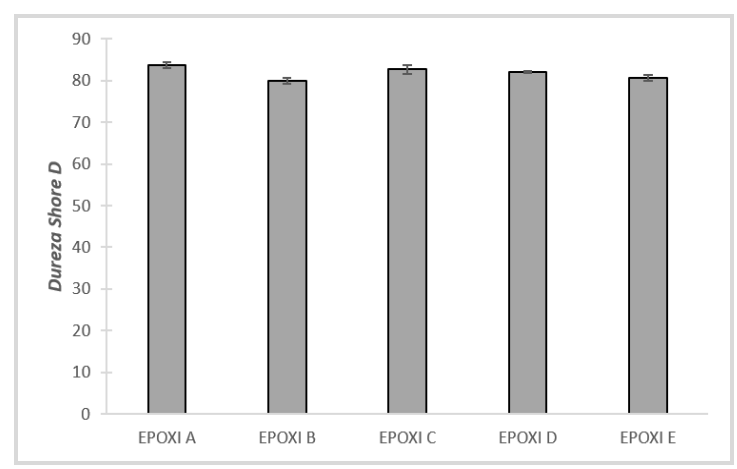

Figura 7: Mediciones de dureza.

Los resultados del análisis multicritério TOPSIS son presentados en el gráfico de la Figura 8, donde se observa la valoración obtenida por cada resina en referencia a su uso en conectores de ductos flexibles. La valoración de la resina $\mathrm{C}$ es un $23,78 \%$ superior a su inmediata antecesora que es la resina $\mathrm{A}$.

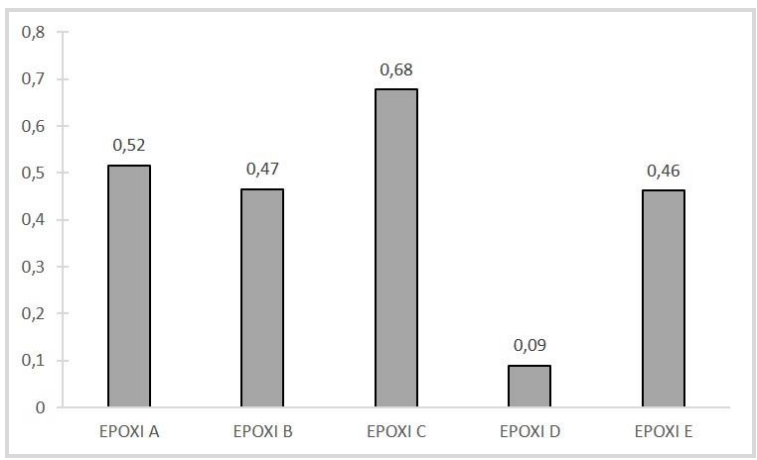

Figura 8: Análisis TOPSIS.

\section{DISCUSIÓN}

Comparando los valores medios de la tensión compresiva en el límite elástico y la tensión compresiva para la ruptura, se encuentran valores bastantes disimiles entre las diferentes resinas evaluadas. Esto bien podría darse por la adición de cargas en partículas que generalmente aumenta el módulo de elasticidad en compresión y resistencia a la compresión, por el aumento de su rigidez [15], como en el caso de la resina A y B. Esta última contiene carga de aluminio y se puede observar que presenta un elevado valor de resistencia a la tensión compresiva de ruptura.

En cuanto al desempeño de las resinas siendo evaluadas en cizallamiento, se observa un comportamiento similar al caso anterior, por el hecho de que la resina A y B contienen cargas de tipo mineral y de aluminio respectivamente y presentan valores medios de tensión de cizalla más elevados que las demás. Aunque en este caso la diferencia no es tan pronunciada. Según PETRIE [15] el contenido de cargas puede contribuir a incrementar la resistencia a las solicitaciones mecánicas de cizalla.

Los resultados de las cinco resinas diferentes obtenidos en los ensayos de arrancamiento muestran que tanto la resina A, B y E tienen un desempeño similar entre sí. Lo que no se corresponde con la resina $\mathrm{C}$ que presenta un valor medio de tensión por adhesión superior a las demás, mostrando una prestación superior como adhesivo frente al substrato utilizado, que es el acero de armadura de tracción de ductos flexibles. Finalmente la resina $\mathrm{D}$ es la que menos tensión soporta en los ensayos de arrancamiento.

Los valores medios de dureza de las cinco resinas evaluadas no presentan diferencias sustanciales, lo que evidencia que en este caso la dureza no es un factor determinante para la selección del material epóxido.

La valoración de las cinco resinas epoxi a través del análisis TOPSIS, permite ver que las resinas A, B y E tienen una performance similar en cuanto a su aplicación para los sistemas de anclaje de los ductos flexibles. No es así para la resina $\mathrm{D}$, que su valoración es baja en comparación a las demás por lo que no se recomienda su uso en esta aplicación. Finalmente la resina $\mathrm{C}$ presentó el mejor desempeño en la evaluación TOPSIS, este hecho se debe principalmente a sus excelentes prestaciones como adhesivo en la junta formada con el acero. 


\section{CONCLUSIONES}

Las resinas epoxi presentan una amplia gama de aplicaciones en la industria, principalmente gracias a la facilidad para variar sus propiedades. Estas variaciones se logran a través de la adición de agentes modificadores, entre los que se pueden nombrar: diluyentes, cargas y pigmentos. Las 5 resinas estudiadas se diferencian entre sí por el tipo y cantidad de contenido de estos agentes modificadores, entre otras cosas. Algunos de los polímeros utilizados en este estudio se utilizan comercial o experimentalmente en sistemas de anclaje, los resultados obtenidos en la matriz de ensayos permiten inferir que dentro de la gama de resinas aplicables existen grandes diferencias entre sus propiedades. Este hecho define la importancia de una minuciosa selección del material utilizado, contemplando que las propiedades de la resina utilizada impactarán directamente en el proyecto de desarrollo de los conectores, a través del sistema de anclaje.

El análisis TOPSIS es una herramienta eficaz para la optimización de selección del material, atendiendo a varios criterios diferentes simultáneamente, en este trabajo permitió determinar la resina que posee el conjunto de propiedades que mejor se ajusta para la aplicación.

\section{AGRADECIMIENTOS}

Los autores de este trabajo quieren agradecer al Laboratório de Metalurgia Física LAMEF/UFRGS por el apoyo y por brindar sus instalaciones para realizar los ensayos.

\section{BIBLIOGRAFÍA}

[1] MORAIS, J. M., Petróleo em águas profundas: uma história tecnológica da PETROBRAS na exploração e produção offshore, 1 ed., Brasília, Instituto de Pesquisa Econômica Aplicada, pp. 83-95, 2013.

[2] API 17B, Recomended Practice for Flexible Pipe, p. 14, 2002.

[3] MUDRY, G. A., Revestimentos de silanos obtidos sobre aço carbono e sua Utilização como agentes promovedores de adesão em uniões aço-resina epóxi, Tesis de M.Sc., PPGE3M/UFRGS, Porto Alegre, RS, Brasil, pp. 28-35, 2011.

[4] DAFLON, M. B., Estudo de adhêrencia de arames da armadura de tração em conectores de dutos flexíveis, Tesis de M.Sc., UERJ/PPGCTM, Nova Friburgo, RJ, Brasil, pp. 34-39, 2010.

[5] CALLISTER, JR., WILLIAM, D. Ciência Engenharia de Materiais - Uma Introdução, 8 ed., Rio de Janeiro, LTC, p. 514, 2012.

[6] ISO 13628-2, Petroleum and natural gas industries - Design and operation of subsea production systems - Part 2: Flexible pipe systems for subsea and marine applications, p. 35, 2000.

[7] TORRES, R.N., TANAKA, R. L., MORINI, R. G., et al., "Flexible pipe anchoring system: resin ratio effects on mechanical properties", In: Proceedings of the ASME 2015 34th International Conference on Ocean, Offshore and Arctic Engineering, OMAE2015-41284, St. John's, Newfoundland, Canada, p. 2, MayJune 2015.

[8] CAMPELLO, G. C., Metodologia de projeto para o sistema de ancoragem de conectores de dutos flexiveis e proposição de nova tecnologia, Tesis de D.Sc., COPPE/UFRJ, Rio de Janeiro, Brasil, pp. 46-48, 2014.

[9] ASTM D695-10, Standard Test Method for Compressive Properties of Rigid Plastics, 2010.

[10] ASTM D732-02, Standard Test Method for Shear Strength of Plastics by Punch Tool; 2002.

[11] KROHLING, R. A., SOUZA, T.T.M., "Dois Exemplos da Aplicação da Técnica TOPSIS para Tomada de Decisão", Revista de Sistemas de Informação da FSMA, n. 8, pp. 31-35, 2011.

[12] XAVIER, F. G., Avaliação da vida em fadiga de um novo modelo de conector para dutos flexíveis Tesis de D.Sc., PPGE3M/UFRGS, Porto Alegre, RS, Brasil, p.p. 86-109, 2009.

[13] CAMPELLO, G. C., CARPIGIANI, M., BERTONI, F., et al., "A Novel Concept of Flexible Pipe End Fitting: Tensile Armor Foldless Assembly", In: Proceedings of the ASME 201231 st International Conference on Ocean, Offshore and Arctic Engineering, OMAE2012-8351, Rio de Janeiro, July 2012.

[14] OLAF, O., TANAKA, R. L., MORINI, R. G., et al., "Thermal effects on the anchoring of flexible pipe tensile armors", In: Proceedings of the ASME 2015 34th International Conference on Ocean, Offshore and Arctic Engineering, OMAE2015-41284, St. John's, Newfoundland, Canada, p.p. 3-6, May-June 2015.

[15] PETRIE, E. M., Epoxy Adhesive formulations, 1 ed., New York, McGraw-Hill, pp. 155-182, 2006. 